\title{
Evaluierung dermatologischer Internetseiten
}

\section{Evaluation of Dermatological Internet Sites}

\section{Zusammenfassung}

Auch in der Medizin findet das World Wide Web als Medium zur Verbreitung von Informationen eine breite Anwendung. Der tatsächliche Nutzwert der Informationen ist dabei sehr unterschiedlich. Seit über zwei Jahren werden in der „Aktuellen Dermatologie“ regelmäßig Internetseiten vorgestellt, die von Interesse für dermatologische Aus- und Weiterbildung sind. In der vorliegenden Arbeit wurden diese Bewertungen zusammengefasst und untersucht. Es zeigte sich bei den untersuchten Punkten „Relevanz der Inhalte für die ärztliche Aus- und Weiterbildung“, „Aktualität“, „Gestaltung und Aufbau der Internetseite“ sowie „grafische Umsetzung des Konzepts“ ein hohes Niveau der vorgestellten Inhalte. Kritisch anzumerken ist jedoch, dass es sich sowohl bei der Auswahl der untersuchten Seiten als auch bei der Einschätzung der Seiten selbst um subjektive Kriterien bzw. Einschätzungen handelt.

\section{Abstract}

In medicine the World Wide Web has become a highly accepted media for spreading information. The benefit of this information for medical education has a broad variability. For more than two years now the „Aktuelle Dermatologie“ publishes internet sites that are of interest for dermatological education. In this paper we summarise the assessment of these pages. The investigated points of interests were „relevance of the content for dermatological education“, „actuality“, „arrangement and structure of the site“ as well as „graphical representation of the concept“. We found a high quality of these investigated items. It has to be pointed out critically that in the selection of the investigated internet site as well in the rating a subjective influence can not be avoided.

auch die Information für Ärzte und Patienten gleichermaßen [1].

Der Informationsanbieter wird dabei von stark divergierenden Ansichten über die Komplexität der Inhalte konfrontiert. Er soll sowohl die Nachfrage nach allgemeinen Informationen befriedigen als auch wissenschaftliche Darstellungen für medizinische Fachgruppen liefern.

Medizinische Informationen werden im Internet von einer Vielzahl von Anbietern dargestellt [2]. So bieten Universitäten, Kliniken, Institute, Selbsthilfegruppen, (Internet)-Zeitungen, Vereinigungen, Firmen, Privatpersonen sowie Gesundheitsportale Informationen zu medizinischen Themen an.

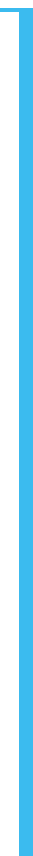

mer größere Verbreitung. Dabei ist sowohl eine Nutzung Infrastrukturen zum Informationsaustausch zwischen den ver-

schiedenen Trägern der Patientenbetreuung gemeint aber
Das Internet spielt in der Entwicklung zur Wissensgesellschaft eine Schlüsselrolle. Dabei hat der technische Teilbereich de gefunden. Dieses Medium bietet die Möglichkeit, Inhalte strukturiert und grafisch ansprechend aufbereitet dem Nutzer zur Verfügung zu stellen. Dabei decken die Inhalte des WWW mittlerweile alle Lebensbereiche $\mathrm{ab}$.

Auch in der Medizin findet die Information via Internet im- 


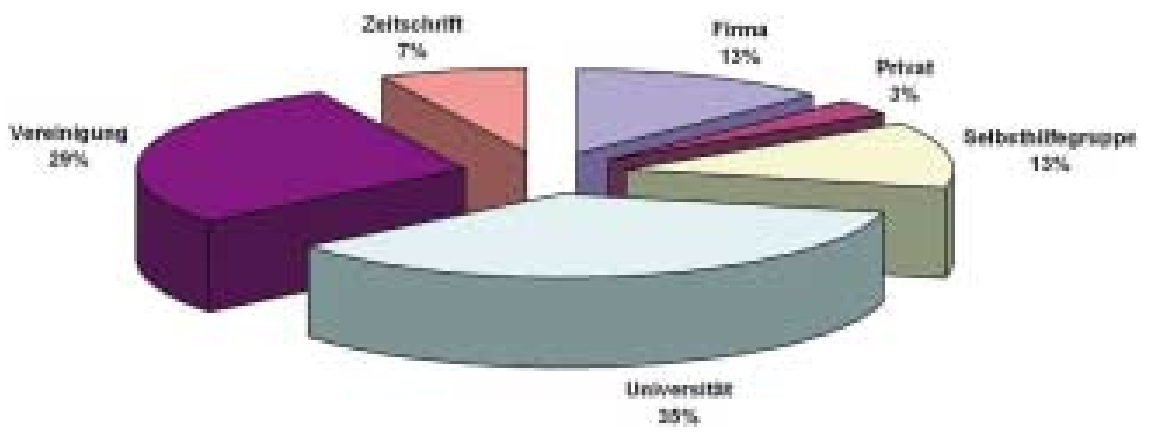

Abb. 1 Hauptgruppen von Institutionen, die dermatologische Inhalte auf Internetseiten anbieten.
Dem Besucher ist es nur schwer möglich, schnell und einfach die Zielgruppe der Informationen zu erkennen sowie die sehr unterschiedliche Qualität der angebotenen Inhalte [3] einzuschätzen, die Validität der Informationen zu prüfen, deren Aktualität zu bestimmen.

In der Zeitschrift „Aktuelle Dermatologie“ erscheinen seit 1998 regelmäßig Beurteilungen dermatologischer Internetseiten, die dem Leser - und damit dem Fachpublikum - eine Einschätzung der betrachteten Informationen auf der jeweiligen Webseite liefern. Diese wurden im Rahmen der vorliegenden Arbeit ausgewertet.

\section{Methoden}

In der Untersuchung wurden retrospektiv die Beurteilungen von insgesamt 70 überwiegend dermatologischen Internetseiten betrachtet und deskriptive statistische Untersuchungen vorgenommen. Dabei wurden alle Beurteilungen im Zeitraum von 1/1998 bis $12 / 2000$ erfasst.

Neben einer Beschreibung der Inhalte, der Zielgruppen, der Funktionalität und einem Kommentar wurde in den Beurteilungen zu jeder Seite eine Einschätzung anhand standardisierter Kriterien vorgenommen.

Die Einschätzungskriterien der Internetseiten beinhalteten die Relevanz für die dermatologisch-fachärztliche Tätigkeit, die Aktualität des Inhalts, die Gestaltung bzw. den Aufbau der Webseite sowie die grafische Aufbereitung des Inhalts. Die oben angegebenen Kriterien wurden auf einer vierstufigen Skala ( 0 - 3 Sterne) beurteilt.

Ein weiteres untersuchtes Merkmal bezog sich auf den Anbieter der Informationen. Dabei erfolgte bei der Auswertung eine Einteilung in folgende Gruppen: Universitäten und Kliniken, Vereinigungen und Organisationen, Selbsthilfegruppen, Firmen, Privatpersonen und Zeitschriften und online-Journale.

\section{Ergebnisse}

Bei der Betrachtung der sprachlichen Ausrichtung der Inhalte zeigte sich, dass eine deutliche Dominanz englischsprachiger Seiten bestand. So wurden 51 (73\%) rein englischsprachige Seiten, 8 rein deutschsprachige (11\%), 6 deutsche und englische Seiten $(9 \%)$ beurteilt. Daneben bot eine Seite die Informationen auf
Englisch und Italienisch, eine Seite auf Englisch und Spanisch, eine Seite auf Englisch und Französisch sowie 3 Seiten dreisprachige und eine Seite viersprachige Informationen. Somit können mit Kenntnissen der englischen Sprache knapp 90\% der untersuchten Internetseiten zum Informationserwerb genutzt werden - mit Kenntnissen allein in Deutsch hingegen nur 20\%.

Bei der Untersuchung, welche Anbieter die Informationen bereitstellen, zeigte sich folgende Anbieterstruktur: 25 Seiten stammten von Universitäten und Kliniken (35\%), 20 Seiten von Organisationen und Vereinigungen (29\%), 9 Seiten von Selbsthilfegruppen (13\%), 9 von Firmen (13\%), 7 von online-Journalen und Zeitungen (7\%) sowie 2 Seiten von Privatpersonen (3\%) (Abb.1).

Bei der Bestimmung der Relevanz für die dermatologische Ausbildung wurde der Mittelwert mit 2,3 $\pm 0,7$ (Mittelwert \pm Standardabweichung) bestimmt. Für die Aktualität betrug der Mittelwert 2,5 $\pm 0,7$. Die Gestaltung bzw. der Aufbau wurde durchschnittlich mit 2,3 $\pm 0,7$ bewertet. Die grafische Aufbereitung der Inhalte wurde durchschnittlich mit 2,2 $\pm 0,6$ bewertet (Abb. 2-5).

\section{Diskussion}

Bei der Betrachtung der sprachlichen Ausrichtung der dermatologischen Internetangebote fiel die Dominanz englischsprachiger Inhalte auf. Dabei ist zu bedenken, dass die Internetseiten unter dem Aspekt der Relevanz für dermatologische Fachkreise ausgewählt wurden. Da medizinische Fachtexte auch in konventionellen Medien bzw. Publikationen überwiegend in englischer Sprache verfasst sind, ist es nicht verwunderlich, dass analog auch bei den Internetangeboten eine Dominanz englischsprachiger Quellen zu verzeichnen ist. Daraus lässt sich im Umkehrschluss nicht sicher ableiten, dass für Patienten Internet-Informationen zu dermatologischen Fragestellungen in deutscher Sprache nicht ausreichend vorhanden sind.

Bei der Bewertung der Internetseiten in der „Aktuellen Dermatologie“ sollte bedacht werden, dass es sich dabei nicht um eine repräsentative Stichprobe aller dermatologischen Internet-Informationsquellen handelt, sondern eine Auswahl durch den Reviewer selbst erfolgt ist. Auch die Beurteilung selbst stellt, besonders bei der Einschätzung von gestalterischen Aspekten, immer nur die subjektive Einschätzung des Einzelnen dar. 


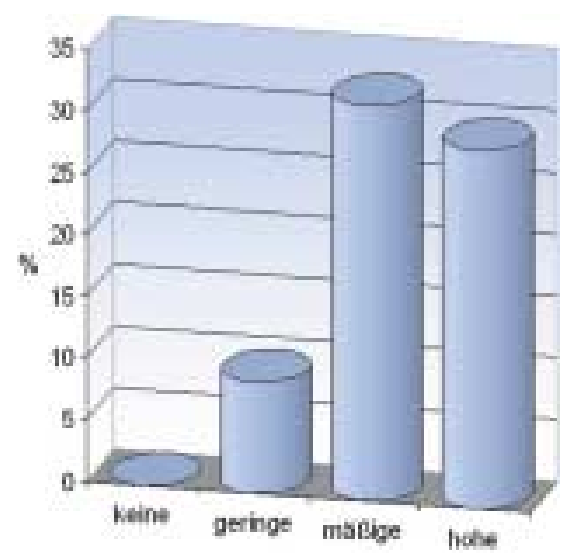

Abb. 2 Relevanz der Internetseiten für die dermatologische Ausund Weiterbildung.

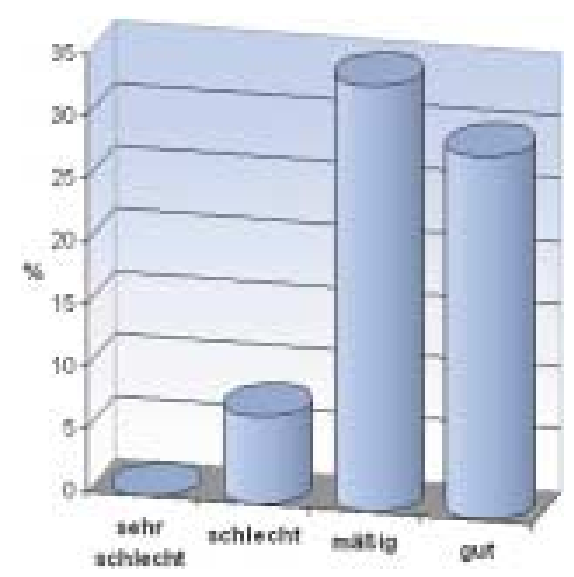

Abb. 4 Einschätzung von Gestaltung und Aufbau der Internetseiten.

\section{Bei der Betrachtung der Relevanz der Informationen für die fach- ärztliche Aus- und Weiterbildung zeigte sich ein erfreulich hohes Niveau von durchschnittlich 2,3 Punkten auf der Skala von 0-3 Punkten. Bei der Beurteilung dieses Wertes muss aber kritisch bedacht werden, dass durch die Autoren der Bewertung eine Vor- auswahl erfolgt ist - und vorwiegend dermatologische Internet- seiten vorgestellt wurden.}

Auch die Einschätzung der Aktualität zeigt ein zum Beobachtungszeitpunkt erstaunlich hohes Maß und wurde mit durchschnittlich 2,5 Punkten bewertet. Dabei stellt die Aktualität der Informationen ein nicht zu unterschätzendes Kriterium dar, unterliegen medizinische Erkenntnisse doch einem stetigen Wandel. Hierbei sollte aber nicht außer Acht gelassen werden, dass möglicherweise Internetseiten zur Darstellung in der „Aktuellen Dermatologie“ ausgewählt wurden, weil sie neu auf dem Markt aufgetaucht sind oder einer generellen Überarbeitung unterzogen wurden. Die Angaben unterliegen außerdem einer deutlichen Inkonstanz. Bei der stichprobenhaften Nachkontrolle von vor zwei Jahren noch als „aktuell“ bezeichneter Internetangebote konnten Seiten gefunden werden, die seitdem keine weitere Überarbeitung oder inhaltliche Weiterentwicklung erfahren haben.

Bei der Gestaltung und Aufbau bzw. der grafischen Umsetzung konnte ein Mittelwert von 2,3 bzw. 2,2 Punkten ermittelt werden. Dabei ist aber kritisch anzumerken, dass die Kriterien zur Bewertung dieser Merkmale einem Wandel in den letzten Jahren unterlegen sind. So sind auf aktuellen Webseiten Darstellungs-

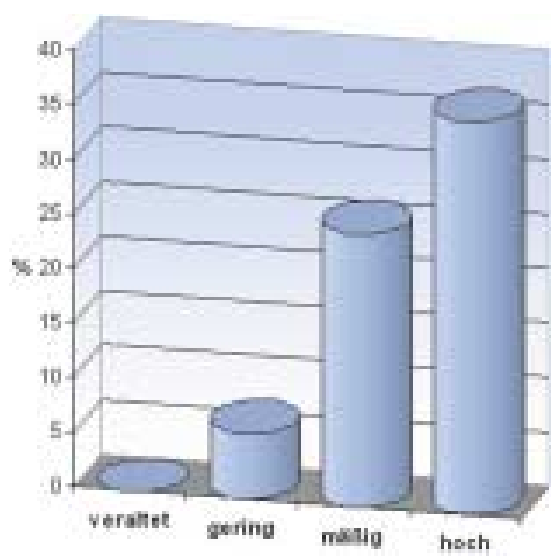

Abb. 3 Einschätzung der Aktualität der Internetseiten.

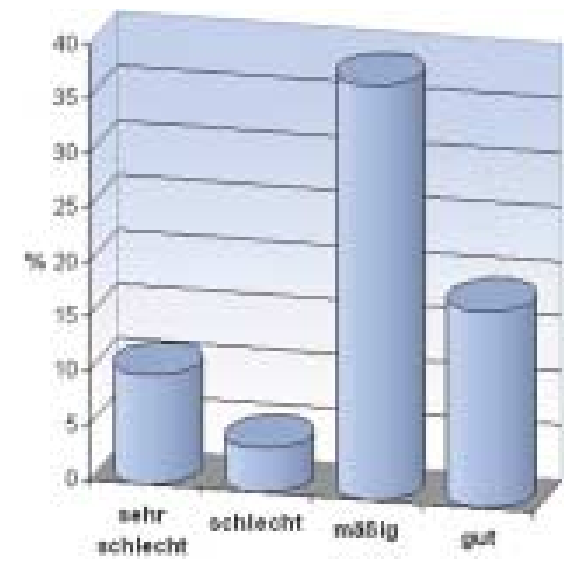

Abb. 5 Einschätzung der grafischen Umsetzung der Inhalte.

möglichkeiten gegeben, die vor wenigen Jahren noch nicht realisierbar waren. Daher würden Seiten, deren Design noch vor zwei Jahren ein sehr gutes Urteil erhalten hätten, heute möglicherweise eine schlechtere Bewertung erhalten.

Die Untersuchung zu den Hauptanbietern von dermatologischen Informationen im Internet ergab, dass jeweils rund ein Drittel der Angebote aus Universitäten bzw. Kliniken und von medizinischen Organisationen und Vereinigungen stammen. Auch wenn dies keine Rückschlüsse über die Qualität und Validität der Inhalte zulässt, so handelt es sich in den meisten Fällen um Informationen von Fachgremien bzw. Fachärzten, so dass eine wissenschaftliche Darstellung der Inhalte und Angebote erfolgt.

Darüber hinaus soll - wie wiederholt diskutiert $[1,4,5]$ - die Forderung erhoben werden, für frei verfügbare Inhalte Qualitätsstandards aufzustellen, deren Durchsetzung zu dokumentieren und auch zu kontrollieren. Dabei wäre wie von Eysenbach und Diepgen vorgeschlagen [6] ein Reviewer-Verfahren ähnlich der medizinischen Fachjournals vorstellbar. Beim med-PICSProjekt (http://www.w3.org/PICS) bzw. medCertain-Projekt (http://www.medcertain.org) agieren Personen, Gruppen, Organisationen oder Unternehmen als so genannter "rating service“. Die von diesen Einrichtungen für ein spezifisches Dokument oder Dokumentengruppen (web-Präsenz) erstellten „Label“ werden dann bei so genannten „Label-Bureaus“ vorgehalten, wobei die Authentizität des Dokuments (passt das Label zum vorliegenden Dokument?) über eine kryptografische Funktionalität (hashFunktion) sichergestellt werden kann. Wenn ein Benutzer eine 
Seite aufruft, werden vom „Label-Bureau“ vergebene Label angefordert und dem Benutzer angezeigt oder auch Filterregeln angewendet, die bestimmte Inhalte ausschließen $[7,8]$.

Andere Systeme wie z.B. die Health On the Net Foundation (www.hon.ch) basieren auf einem Gütesiegel, was aber nicht die Zertifizierung der Seite zur Basis hat, sondern welches der Betreiber einsetzen kann, wenn er sich mit einem bestimmten Kodex einverstanden erklärt. „Insgesamt ist die gesamte Initiative so zu bewerten, als wenn man Autofahrern vorschlagen würde, sich eine TÜV-Plakette selbst aufs Auto zu heften, wenn sie meinen, dass mit ihrem Auto alles in Ordnung ist"[9].

Zusammenfassend lässt sich festhalten, dass die in der „Aktuellen Dermatologie“ dargestellten dermatologischen Internetangebote ein hohes Maß an Aktualität und klinischer Relevanz haben und auch unter gestalterischen Aspekten ansprechend sind. Daher kann der Besuch dieser Seiten einen sinnvollen Beitrag zur ärztlichen Aus- und Weiterbildung leisten, dem Arzt aber auch Anhalt dafür sein, seine Patienten gezielt auf qualitativ hochwertige Internetangebote aufmerksam zu machen. Es bleibt jedoch zu bedenken, dass die Darstellungen und Bewertungen von Internetseiten in der „Aktuellen Dermatologie“ immer eine stark subjektive Komponente aufweisen, sich der Leser daher immer selbst ein Bild der angebotenen Inhalte machen sollte.

\section{Literatur}

${ }^{1}$ Wainwright BD. Clinically relevant dermatology resources and the Internet: An introductory guide for practicing physicians. Dermatol Online J 1999; 5: 8

${ }^{2}$ Murphy KR. Computer-based patient education. Otolaryngol Clin North Am 1998; 31: 309-317

${ }^{3}$ McClung HJ, Murray RD, Heitlinger LA. The Internet as a source for current patient information. Pediatrics 1998; 101: 2

${ }^{4}$ Jadad AR, Gagliardi A. Rating health information on the Internet: navigating to knowledge or to Babel? JAMA 1998; 279: 611 -614

${ }^{5}$ Silberg WM, Lundberg GD, Musacchio RA. Assessing, controlling, and assuring the quality of medical information on the Internet: Caveant lector et viewor - Let the reader and viewer beware. JAMA 1997; 277: $1244-1245$

${ }^{6}$ Eysenbach G, Diepgen TL. Labeling and filtering of medical information on the Internet. Methods Inf Med 1999; 38: 80-88

${ }^{7}$ World Wide Web Consortium, Platform for Internet Content Selection (PICS): http://www.w3.org/PICS/; 21-8-2001

${ }^{8}$ Eysenbach G, Köhler C, Yihune G, Lampe K, Cross P, Brickley D. A metadata vocabulary for self- and third-party labeling of health web-sites. Proc AMIA Annu Fall Symp 2001: in press

${ }^{9}$ FAQ Cybermedizin, Eysenbach G. http://www.yi.com/home/EysenbachGunther/faq.htm; 21-8-2001 